\title{
The Multidimensional Construction of Theories on Literary Translation Criticism
}

\author{
Chunhua Shen \\ School of Foreign Languages, Wuhan Polytechnic University, Wuhan, 430028, China \\ email: 594561792@qq.com
}

Keywords: Translation Criticism; Standards of Translation Criticism; Literary Translation Criticism; Multidimensional View

\begin{abstract}
Translation criticism is a significant part of translation science, and plays a valuable role in guaranteeing translation quality. So, this essay aims to help foster the development of translation criticism in a theoretical, systematic, and comprehensive manner, and enhance the all-round development of translation research.
\end{abstract}

\section{Introduction}

Translation criticism is a major field of translation science, for it is involved in translation practice, development of translation talents, the subject building of translation science and many other relative respects. As the link between translation theory and practice, translation criticism is developed on the basis of both their full development and that of translation standards.

\section{On Translation Criticism}

Translation criticism is a major part of translation research. According to Peter Newmark (1988), translation criticism is a basic link between translation theory and practice. In Ji Xianlin's opinion, translation criticism helps improve and lead the development of translation in the right direction. However, consensus about the definition of translation criticism hasn't been reached among scholars.

The English-Chinese Dictionary of China refers to that as a sort of comprehensive evaluation; translation criticism offers accurate standards to judge the process and quality of translation, and the value of translation works. Huang Qiongying believes that translators can study translation criticism with relative theories, and analyze the translation theory, process and works through correct standards, thus helping improve translators' skill and the quality of translation.

\section{Introduction of Theories about Translation Criticism}

\section{The Systems of Translation Criticism}

\section{1) Connotation}

As translation criticism is a systematic and interrelated academic discourse, the construction of the system of translation criticism will help people study translation better in a more comprehensive and organized way, and also with a perspective throughout the ancient and present. Besides, the objects to be researched and dealt with will be regarded as a whole. The system's structure and function will be analyzed, and the relation among the system, factors, and the environment will be studied, as well as the regularity of their changes, which are aimed to help people gain a complete knowledge of the system's characteristics and regularity, better adjust these relations, and get the system's existence and development well meet practical needs. Actually, that is what the system theory basically means. The system of translation criticism, therefore, can be regarded as an organic whole, combined with a few factors in a certain form and enjoying certain functions: These factors don't exist independently; instead, each of them functions in its own way. Its overall function also has features of other systems, including integrity, relation, hierarchy structure, dynamic balance, and time sequence regularity. 


\section{2) Function}

As a systematic knowledge structure, translation criticism mainly functions in three aspects. When comparison happens between different versions of translation results, the system can help throw light upon reasons behind them.

The system of translation criticism can help people get a macro knowledge of the history and present situation of translation, and enable people to consider and explain problems from the perspective of culture: Why are certain topics of translation often or seldom illustrated? Why does some of them gain dominance but lose it later? What kind of ideology, value, and culture are these phenomena shaped by? To cite an example here, during the first decade of the 20th century, the ruling science of poetry in Europe and America was strongly against Ezra Pound's ideas, for he believed that to be a significant part of the currency, poetry must be endowed with life and images, get rid of hackneyed and stereotyped expressions and rigid forms. Later due to the efforts and persistence of imagistic poets like Ezra Pound, imagistic poetry had succeeded to reach the central position from the edge in the literature of the target language.

The system of translation criticism can be used to explore a specific problem of translation. Translation critics can get translation criticism classified and analyzed according to a certain functional type of text(informative, expressive, or appealing type) and reader group, and can also conduct research by combining different linguistic and non-linguistic factors with various types of translation criticism (Reiss, 2004). As a knowledge structure, the system of translation criticism should observe the basic philosophy of the system theory that all parts should defer to the entire translation text, and quality of translation should be judged with all relative factors considered.

The system of translation criticism is helpful to dynamic research of a series of translation problems. Dynamic standards of translation criticism can help people better understand the translation field and make the features of interlingual translation more distinctive. This sort of theory concerns the description of dynamic process, involves both value and in-value judgments, and also enjoys cultural interpretation, so it can be used to correct the deflected direction of translation criticism, and even find the idealized one.

In brief, the systematic translation criticism can be employed in macro, micro, and dynamic research on a train of translation problems.

\section{Standards of Translation Criticism}

Just as translation standards are key issues of translation theory, standards of translation criticism are also quite important to translation criticism.

1) The Present Situation of Research

Having considered various translation theories in both the national and western history, Wen Xiuying concluded in her book Translation Criticism: From Theory to Practice, "A rather contradictory thing seems to have been true in the translation field: On the one hand, unified standards of translation criticism are considered not to exist; on the other, we're trying to find out such standards." The same issue has been referred to by Yang Xiaorong in his book An Introduction to Translation Criticism. He believes that our traditional translation theory has much to do with standards of translation criticism. Moreover; translation research lacks novel ideas and perspectives, for it is mostly limited in literary translation.

2) The requirements

As Yang Xiaorong puts it, "No matter what theories they are, the theories of faithfulness, expressiveness, and elegance, similarity in spirit, sublimity, or equivalence theory, each of them has been put forward with its own background and conditions. Without understanding their true meaning by reading source texts, it will be hard to make persuasive comments. Likewise, it is also unscientific to judge translation texts with various standards mixed. So it is necessary for us to seek new perspectives and pluralistic standards on the basis of a scientific and comprehensive understanding of the present ones, find out their characteristics, and put forward new requirements.

Objectivity: This is an idea which seems contradictory but actually not: It requires that critics should hold an objective attitude rather than rely on his own subjective feelings; the standards should have a scope of application, not just for a certain translation text, translator, and reader. 
Scientificity: Standards of translation criticism must be scientific, which means it should be justified, practical, and easy to understand.

Pluralism: The philosophy of plural complementation was introduced by Gu Zhengkun a few years ago. In his opinion, diversification is not being full of variety, but the pursuit of finiteness in unlimitedness. Pluralistic standards are complementary, and one standard's advantage may be the disadvantage of another.

Dynamics: Since things are in consistent development, success happens only when problems are treated in the same angle of development. Dynamics matters much, and standards should follow the pace of time and enjoy history matching to avoid rigid translation criticism.

Relation: Translation criticism should take into account the historical context of translation works, and avoid criticizing them with standards from a different time. That's to say, "standards of translation criticism should enjoy historical relation" (Wen Xiuying, 2007). Additionally, the standards should also have objective relation, meaning that classification and nature of critical objects should also be considered.

\section{3) Several Standards}

Since traditional standards of translation criticism fail to get rid of static and one-dimensional mindsets, then new standards should manage to do this. The making of standards of translation criticism should get the following factors involved.

Firstly, combine criticism on translation texts with that on translators. Translation criticism involves both the comparative analysis of texts and the subjective role of translators. When there are striking differences between source and translation texts, we can try to make analysis based on translators' standpoint and purposes.

Secondly, combine practical and theoretical criticism. People who do translation practice often ignore the work of those who make translation research, thus making their criticism lack systematic and theoretical properties. So the right way is to combine practical and theoretical criticism.

Thirdly, combine macro and micro criticism. Translation criticism should pay attention not only to the analysis of diction and sentence structures, but also to the comparison of the style, linguistic features and aesthetic value of translation texts.

Lastly, combine cultural and linguistic criticism. Thanks to cultural turn, most people believe it is the trend to concern about culture instead of language, while those who stick to linguistic research consider culture is just too general. Actually, translation criticism will be more persuasive if cultural and linguistic criticism goes hand in hand.

In conclusion, standards of translation criticism mean so much to the development of translation criticism. Limited by some conditions, the four standards mentioned above are certainly not the only ones. Correct standards should be novel, scientific and based on relative translation theories and practice.

\section{On Literary Translation Criticism}

Literary translation criticism is a new subject, which occupies a valuable position in translation research. It is not only literary and aesthetic, but also scientific and research-oriented. However, there is still a long way to go for its theoretical development. Both the traditional and modern patterns of translation criticism, like the perception-oriented pattern and de-constructivism, are either too macro or micro.

1) Basic Concepts of Literary Translation Criticism

The first basic concept of literary translation criticism is its definition, which includes general and specific aspects. Literary translation criticism is usually the focus of traditional Chinese translation criticism. General translation criticism refers to the process of interpreting and judging translation, involving the source and translation texts, the translator, reader, and social and cultural acceptance. And specific translation criticism focuses on rational thinking, and requires detailed analysis of both the source and translation texts. Comparatively, general translation criticism can better reflect the nature of translation criticism.

The second basic concept is the fundamental rules of literary translation criticism. As the major measuring dimension of literary translation practice and research, literary translation criticism is the 
medium between translation theory and practice. The prosperity of translation cannot happen without the promotion of translation criticism, and literary translation criticism aims to increase the quality of translation, and foster the development of both the translation theory and practice. Given this purpose, literary translation criticism should be objective, constructive, and dynamic.

2) Objects of Literary Translation Criticism

In general, philosophy about this aspect is not rational enough. Firstly, in our country's traditional practice of literary translation criticism, there is a superficial trend in understanding texts, for finding faults with texts seems to have become the purpose of literary translation criticism. Secondly, the continual development of modern translation theory makes scholars aware of that translation is absolutely not a closed process just from one text to the other. If literary translation criticism only focuses on translation results, then one-sided comments will be resulted in.

But under this sense has the current literary translation criticism gone too far from texts, and have descriptive, sociological and cultural translation criticism become rather dominant. In fact, literary translation is basically an art of bi-linguistic shift, with the plot, main idea and the style of the translation text limited by the source text. And translation criticism mostly values the transmission of ideas, images and expressions between these two sorts of texts as well as reasons behind these differences. So in this sense, texts should exactly be the major focus of translation criticism.

3) Methods and Phases of Literary Translation Criticism

In Encyclopedia Logic, Hegel pointed out, "Method is not external form, but the soul of the content and concepts." So it can be reached that methods of research are dependent on and even decided by its content. Scholars both at home and abroad have done much to explore methods of translation criticism, including Newmark's method of functional criticism and analytical method of criticism, Xu Jun's six methods: logical verification method, quantitative and qualitative analysis method, semantic analysis method, sampling analysis method, comparison of different translation versions, and translation appreciation method, and the four methods put forward by Fang Mengzhi, comparison, inquiry, description, and demonstration.

These examples also show that agreement hasn't been reached about specific methods of translation criticism. Nevertheless, it is still practical for us to make a general and guiding definition. The scientific aspect of literary translation lays emphasis on the regularity of translation process, while the artistic aspect attaches importance to the creativity. Considering these two kinds of features, methods of translation criticism should focus on both accuracy and logical deduction, respectively belonging to intra-linguistic and inter-linguistic analysis. The premier emphasizes textual analysis and linguistic interpretation; the latter values factors beyond texts, like society, culture and readers. The two methods are not contradictory but interdependent.

\section{Conclusion}

With the continual development of research on translation theory, translation criticism is also enjoying a favorable development trend. The system of translation criticism has been built and getting improved, research on its standards is becoming deeper and richer, and literary translation criticism is also undergoing prosperity, all of which have helped foster the development of the theory of translation criticism.

In general, not only does translation criticism provide methods and standards for translation practice, but also it opens the door to a more profound exploration of translation. Greater attention should be paid to translation criticism; if not, translation will fail to be integrated.

\section{References}

[1] Halliday, M. A. K. \& Hasan, R. Language, Context and Text [M]. Victoria: Deakin University Press, 1985.

[2] Hickey, L. The Pragmatics of Translation [M]. Shanghai Foreign Language Education Press, 
2001.

[3] Katharina R. Translation Criticism: The Potentials\&Limitations [M]. St. Jerome Publishing, 2000.

[4] Newmark, Peter. About Translation [M]. London: Multilingual Matters Ltd, 1991.

[5] Nida, Eugene A. Language and Culture-Contexts in Translating [M. Shanghai: Shanghai Foreign Language Education Press, 2001.

[6] Reiss, Katharina. Translation Criticism: The Potentials \& Limitations [M]. Shanghai: Shanghai Foreign Language Education Press, 2004. 